\title{
Disorders of Consciousness in Hospitalized Patients with COVID-19: The Role of the Systemic Inflammatory Response Syndrome
}

Amelia K. Boehme1, Kevin Doyle², Kiran T. Thakur ${ }^{3}$, David Roh², Soojin Park², Sachin Agarwal2, Angela G. Velazquez ${ }^{2}$, Jennifer A. Egbebike², Caroline Der Nigoghossian ${ }^{4}$, Morgan L. Prust ${ }^{2}$, Jon Rosenberg ${ }^{2}$, Daniel Brodie ${ }^{5}$, Katherine N. Fishkoff ${ }^{6}$, Beth R. Hochmann ${ }^{6}$, Leroy E. Rabani ${ }^{7}$, Natalie H. Yip ${ }^{5}$, Oliver Panzer ${ }^{8}$ and Jan Claassen ${ }^{2^{*}}$ (i)

\begin{abstract}
Background: Prevalence and etiology of unconsciousness are uncertain in hospitalized patients with coronavirus disease 2019 (COVID-19). We tested the hypothesis that increased inflammation in COVID-19 precedes coma, independent of medications, hypotension, and hypoxia.

Methods: We retrospectively assessed 3203 hospitalized patients with COVID-19 from March 2 through July 30, 2020, in New York City with the Glasgow Coma Scale and systemic inflammatory response syndrome (SIRS) scores. We applied hazard ratio (HR) modeling and mediation analysis to determine the risk of SIRS score elevation to precede coma, accounting for confounders.

Results: We obtained behavioral assessments in 3203 of 10,797 patients admitted to the hospital who tested positive for SARS-CoV-2. Of those patients, 1054 (32.9\%) were comatose, which first developed on median hospital day 2 (interquartile range [IQR] 1-9). During their hospital stay, 1538 (48\%) had a SIRS score of 2 or above at least once, and the median maximum SIRS score was 2 (IQR 1-2). A fivefold increased risk of coma (HR 5.05, 95\% confidence interval 4.27-5.98) was seen for each day that patients with COVID-19 had elevated SIRS scores, independent of medication effects, hypotension, and hypoxia. The overall mortality in this population was $13.8 \%(n=441)$. Coma was associated with death (odds ratio 7.77, 95\% confidence interval 6.29-9.65) and increased length of stay (13 days [IQR 11.9-14.1] vs. 11 [IQR 9.6-12.4]), accounting for demographics.
\end{abstract}

Conclusions: Disorders of consciousness are common in hospitalized patients with severe COVID-19 and are associated with increased mortality and length of hospitalization. The underlying etiology of disorders of consciousness in this population is uncertain but, in addition to medication effects, may in part be linked to systemic inflammation.

Keywords: COVID-19, Disorders of consciousness, Coma, Inflammation, Systemic inflammatory response syndrome

*Correspondence: jc1439@cumc.columbia.edu

2 Division of Critical Care Neurology, Department of Neurology, Columbia University Irving Medical Center, New York-Presbyterian Hospital, 177 Fort Washington Avenue, MHB 8 Center, Room 300, New York, NY 10032, USA Full list of author information is available at the end of the article

\section{Introduction}

Novel coronavirus disease 2019 (COVID-19) is a global pandemic caused by severe acute respiratory syndrome coronavirus 2 (SARS-CoV-2). SARS-CoV-2 is associated with high morbidity and mortality, causing an enormous strain on health care systems [1]. Neurological symptoms have been reported in almost half of these patients [2-5] 
and include diseases affecting the central nervous system, such as ischemic and hemorrhagic strokes, venous sinus thrombosis, and endothelialitis. Additionally, the peripheral nervous system may be affected, resulting, for example, in Guillain-Barré syndrome. Underlying mechanisms for neurological complications of SARS$\mathrm{CoV}-2$ are largely unknown but may include postinfectious immune mediated processes, direct viral infection, and hyperinflammatory and hypercoagulable states. Impairment and fluctuating states of consciousness, such as coma and delirium [6], have been reported [7, 8]. In non-COVID-19 patients, coma is known to have a major impact on outcomes and goals of care decisions and is also known to complicate and prolong hospitalizations [9-11]. Prevalence and etiology of coma in patients suffering from COVID-19 remain largely unknown at this time, as concerns for health care worker safety provide challenges for detailed neurological assessments. Systemic inflammatory response syndrome (SIRS), a nonspecific systemic marker of inflammation, has been used previously as a marker of systemic inflammation in studies evaluating the role of systemic inflammation on outcomes in neurological disorders [12-17]. Herein, we report the prevalence of disorders of consciousness in hospitalized patients with COVID-19 using the Glasgow Coma Scale (GCS) and evaluate the role of SIRS on risk of coma in patients with COVID-19.

\section{Methods \\ Patients}

All adult patients (aged $\geq 18$ years old) admitted to the Columbia University Irving Medical Center campus of New York-Presbyterian Hospital meeting Centers for Disease Control and Prevention criteria for definitive diagnosis of COVID-19 [18] from March 2 through July 30,2020 , who had at least one behavioral assessment performed using the GCS were included in this retrospective observational cohort study. The hospital is an approximately 700-bed tertiary referral center located in northern Manhattan, New York City. The number of intensive care unit (ICU) beds was increased from 117 at the beginning of the pandemic to more than 300 at its peak. SARS-CoV-2 infection was diagnosed by nasopharyngeal swab using real-time reverse-transcription polymerase chain reaction (rtRT-PCR) by the New York City Department of Health from March 2 through March 10, 2020, after which testing was performed using rtRT-PCR in the clinical microbiological laboratory of New York-Presbyterian Hospital. We collected data on demographics, obesity (defined as a body mass index $[\mathrm{BMI}] \geq 30$ ), behavioral assessments, disease measures, and treatments from the electronic medical record in all hospitalized patients.

\section{GCS Measures}

Standardized GCS assessments by health care providers were obtained at least daily as part of routine clinical care, particularly in the step-down unit and critical care setting. In the step-down and critical care setting, hourly documentation is available. We collected the GCS [19] score, including subscores, with the primary end point being coma (GCS score of 8 or below). Secondary outcomes included not obeying motor commands (GCS motor response subscore 1-5), lack of verbal response demonstrating orientation (GCS verbal response subscore 1-4), and no verbal response (GCS verbal response subscore 1). Coma was defined as any GCS total score less than or equal to 8 . We focused on the GCS assessments to define coma instead of other, more sophisticated assessments given the availability of these data collected as part of routine clinical care and the inability to obtain established measures, such as the Coma Recovery Scale Revised.

\section{Disease Course}

Inflammation was defined using a nonspecific systemic marker of inflammation, the SIRS score [20], by assigning a value of 1 for each of the following: heart rate $>90$ beats per minute, respiratory rate $>20$ breaths per minute, body temperature $>38{ }^{\circ} \mathrm{C}$ or $<36{ }^{\circ} \mathrm{C}$, and serum white blood cell count $<4000$ or $>12,000 / \mathrm{mm}^{3}$. SIRS scores of 2 or above were considered abnormal. Patients were classified as having an abnormal SIRS score only if the SIRS score elevation occurred prior to coma or prior to discharge in the event of no coma. The SIRS score has been used previously as a nonspecific systemic marker of inflammation to evaluate how systemic inflammation can influence neurologic outcomes [12-17]. Additionally, we recorded mean arterial blood pressure and oxygen saturation over time. Hypoxia was defined as any measure of oxygen saturation below $90 \%$ regardless of the provided oxygen supplementation. Laboratory measures investigated included serum levels of interleukin-6 (IL-6), D-dimer, lactate dehydrogenase, ferritin, creatinine, and creatinine kinase and the absolute lymphocyte count. We recorded the daily presence of medications that are known to affect behavioral assessments (including intravenous or oral sedatives [e.g., midazolam, lorazepam, propofol, chlordiazepoxide, clonazepam], intravenous or oral opioid analgesics [e.g., hydromorphone, fentanyl, oxycodone], and intermittent or continuous intravenous infusion neuromuscular blockers given for the management of acute respiratory distress syndrome (ARDS)) and enable prone positioning during extracorporeal membrane oxygenation (ECMO) and not in the context of intubation (e.g., cisatracurium, rocuronium, vecuronium). Additionally, 
we recorded therapeutics used in COVID-19 treatment, including remdesivir, hydroxychloroquine, and immunomodulatory agents (i.e., IL-6 receptor antagonists and corticosteroids).

\section{Statistical Analysis}

Categorical variables were expressed as numbers and percentages and were compared using Fisher's exact or $\chi^{2}$ tests, as appropriate. Continuous variables were expressed as medians and interquartile ranges (IQRs) or as means and standard deviations, as appropriate, and were compared using Wilcoxon signed-rank tests. Logistic regression models were used to evaluate whether the odds of certain characteristics (e.g., demographics or admission to an ICU) differed between patients with COVID-19 who did and those who did not have behavioral assessments recorded. Kaplan-Meier curves were generated to illustrate the unadjusted time to a behavioral outcome (e.g., onset of coma). Time to event analysis using Cox proportional hazards modeling was used to calculate the association between inflammatory markers and behavioral abnormalities (hazard ratio [HR] and 95\% confidence intervals [CI]). Mediation analyses were conducted to determine the extent to which paralytic or sedative medications, or a combination of paralytic and sedative medications, explained the relationship between SIRS and coma [21]. All tests were two sided at an $\alpha$ of 0.05 . Statistical analyses were performed with $R$ statistical software, version 3.4.1 (R Project for Statistical Computing).

\section{Results}

\section{Patients}

From March 2 through July 30, 2020, we obtained behavioral assessments in 3203 of 10,797 patients admitted to the hospital who tested positive for SARS-CoV-2. Of those included, a total of 1623 (50.7\%) were managed for part or all of their clinical course in an ICU and 1580 $(49.3 \%)$ were treated in a non-ICU setting. The mean age of included patients was $63.2( \pm 17.2)$ years old, 1,788 (55.8\%) patients were men, and 948 (24.6\%) patients had a $\mathrm{BMI} \geq 30$ (Table S1). Medical management included invasive mechanical ventilation in 336 (10.5\%). Patients were excluded if they were not admitted or did not have at least one GCS assessment. Patients in the study cohort with behavioral assessments were more frequently admitted to the ICU and tended to be older compared with hospitalized patients with COVID-19 who were not enrolled $(n=7594)$ (Table S2. Our study found a low prevalence of bacterial sepsis (1.6\%), a finding consistent with other reports [22].

We obtained a median number of 8 GCS assessments per patient (IQR 3-19) and found that 1090 (32\%) patients were comatose (Table S1 and Fig. 1). Approximately $83 \%$ of all GCS assessments and $56 \%$ of GCS assessments greater than 8 were obtained in the ICU setting. Coma first developed on median hospital day 2 (IQR 1-9), and median time to event for noncomatose patients was 6 days (IQR 2-18). Nine hundred sixtyone $(91.2 \%)$ of comatose patients, but only $619(28.8 \%)$ of noncomatose patients, were admitted to the ICU. We obtained daily SIRS scores and found that 1538 (48.0\%) had a score of 2 or above at least once during their hospital stay, which persisted for a median of 1 day (IQR 1-2).

\section{Predictors of Coma}

Patients who became comatose were more likely men. Preceding the onset of coma, they were more likely to have hypoxia and to have received sedatives, paralytics, or opiate medications (Table 1). Ninety-three patients had a GCS score of 9-12 (Table S3). Coma was more common in patients who were intubated (HR 1.68, 95\% CI 1.42-1.99) and those who had an SIRS score of 2 or above at least once during the hospital stay (HR 5.92, 95\% CI 5.03-6.97). Age, patients with a BMI of 30 or above, Black race, Hispanic ethnicity, or hypotension prior to the onset of coma were not associated. Accounting for each day that patients with COVID-19 had an SIRS score

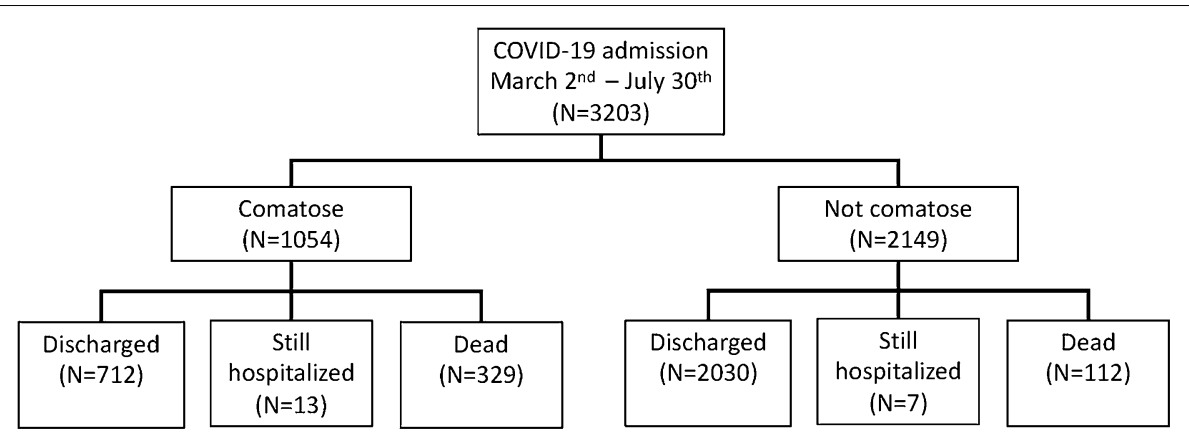

Fig. 1 Flowchart. Coma and outcomes of patients with coronavirus disease 2019 (COVID-19) 
Table 1 Demographic and clinical characteristics measured prior to coma or discharge

\begin{tabular}{|c|c|c|c|c|}
\hline & \multicolumn{2}{|l|}{ Coma } & \multirow{2}{*}{$\begin{array}{l}\text { Univariate } \\
\text { OR }(95 \% \mathrm{Cl})\end{array}$} & \multirow{2}{*}{$\begin{array}{l}\text { Multivariable } \\
\text { OR }(95 \% \mathrm{Cl})\end{array}$} \\
\hline & Comatose $(n=1054)$ & $\begin{array}{l}\text { Not } \\
\text { comatose } \\
(n=2149)\end{array}$ & & \\
\hline \multicolumn{5}{|l|}{ Characteristics } \\
\hline Age $\geq 60$ years & $698(66.2 \%)$ & $1,354(63.0 \%)$ & 1.15 (0.98 to 1.34$)$ & 0.95 (0.82 to 1.09$)$ \\
\hline Male sex & $642(60.9 \%)$ & $1,146(53.3 \%)$ & $1.21(1.06$ to 1.36$)$ & 1.19 (1.04 to 1.37$)$ \\
\hline $\mathrm{BMI} \geq 30$ & $351(33.3 \%)$ & $597(27.8 \%)$ & 1.25 (1.10 to 1.42$)$ & 1.15 (0.99 to 1.33 ) \\
\hline Race, African American or Black & $206(19.5 \%)$ & 437 (20.3\%) & 1.00 (0.86 to 1.17$)$ & 0.90 (0.76 to 1.07$)$ \\
\hline Ethnicity, Hispanic & $343(32.5 \%)$ & $764(35.6 \%)$ & 1.12 (0.98 to 1.27$)$ & 0.94 (0.81 to 1.08$)$ \\
\hline \multicolumn{5}{|l|}{ Hospital course } \\
\hline$I C U^{a}$ & $961(91.2 \%)$ & $619(28.8 \%)$ & $13.9(11.2$ to 17.2$)$ & \\
\hline Intubated & $323(30.6 \%)$ & $13(0.6 \%)$ & 4.89 (4.29 to 5.58$)$ & 1.68 (1.42 to 1.99$)$ \\
\hline Paralytics & $197(18.7 \%)$ & $5(0.23 \%)$ & $3.82(3.26$ to 4.46$)$ & \\
\hline Sedatives & $714(67.7 \%)$ & $219(10.9 \%)$ & 7.57 (6.65 to 8.62$)$ & 3.34 (2.85 to 3.91$)$ \\
\hline Opiates & $212(20.1 \%)$ & $14(0.6 \%)$ & 4.40 (3.61 to 5.36$)$ & 1.09 (0.87 to 1.38$)$ \\
\hline \multicolumn{5}{|l|}{ Vital signs ${ }^{b}$} \\
\hline Oxygen saturation $<90 \%$ on room air at any time prior to outcome & $131(12.4 \%)$ & $94(4.4 \%)$ & 1.73 (1.44 to 2.08$)$ & 1.08 (0.88 to 1.32$)$ \\
\hline Mean arterial blood pressure $<60 \mathrm{~mm} \mathrm{Hg}$ & $29(2.9 \%)$ & $29(1.4 \%)$ & 1.41 (0.97 to 2.04$)$ & 0.98 (0.66 to 1.47$)$ \\
\hline Best GCS score, median (IQR) & $15(11$ to 15$)$ & 15 (15 to 15$)$ & & \\
\hline Worst GCS score, median (IQR) & $3(3$ to 4$)$ & $15(14$ to 15$)$ & & \\
\hline Best RASS score, median (IQR) & $0(0$ to 1$)$ & $0(0$ to 0$)$ & & \\
\hline Worst RASS score, median (IQR) & $-5(-5$ to -4$)$ & $0(-1$ to 0$)$ & & \\
\hline Number of days with a positive CAM-ICU result, median (IQR) & 1 (0 to 4$)$ & $0(0$ to 0$)$ & & \\
\hline
\end{tabular}

Data reported as median (IQR) or no. (\%), as appropriate

$B M I$ body mass index, CAM-ICU confusion assessment method for the intensive care unit, $C I$ confidence interval, GCS Glasgow Coma Scale, ICU intensive care unit, IQR interquartile range, RASS Richmond Agitation-Sedation Scale, SIRS systemic inflammatory response syndrome

a ICU admission was not included in the multivariable model because nearly all coma patients were in the ICU, rendering the model not stable

b Measurements reflect data obtained prior to the onset of coma

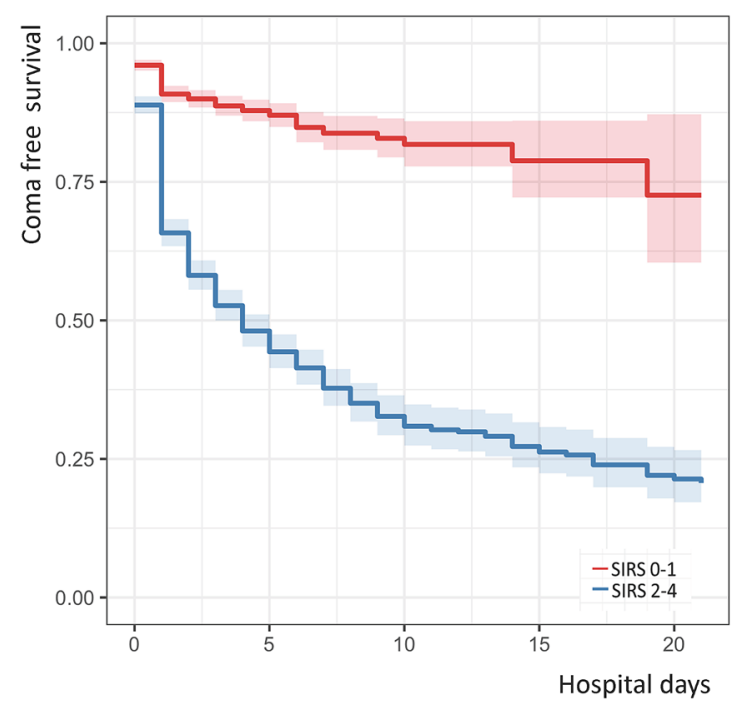

Fig. 2 Systemic inflammation and coma. Development of coma over time stratified by the SIRS score (SIRS score 0-1 vs. SIRS score 2-4). SIRS systemic inflammatory response syndrome of 2 or more, a fivefold increased risk of coma was seen (HR 5.05, 95\% CI 4.27-5.98; Fig. 2). The risk of coma increased with the number of days with an SIRS score of 2 or above for patients receiving neuromuscular blocking agents, those receiving sedative or opiate medications, and those receiving none of these medications (Table 2 and Fig. S1). The lag between SIRS first crossing a threshold of 2 and the onset of coma was 4 days (IQR 1-11). Serum measures of inflammation and disease severity were available, including IL-6, D-dimer, lactate dehydrogenase, ferritin, absolute lymphocyte count, creatinine, and creatinine kinase, which were elevated in patients with coma (Table S4 and Fig. S2). To account for the relationship between SIRS and medications and how this could relate to coma, we explored medications as a mediator between the relationship of SIRS and coma. We found paralytic medications to be a statistically significant mediator $(p<0.0001)$ and found that use of paralytic medications explained $60 \%$ of the effect between SIRS and coma (Table 3). Sedatives and opiates were also statistically significant mediators $(p<0.0001)$, but they had 
Table 2 Models predicting coma, accounting for SIRS score of $\geq 2$ and number of days with a SIRS score $\geq 2$

\begin{tabular}{|c|c|c|c|}
\hline & Coma, $n(\%)$ & $\mathrm{SIRS} \geq 2, \mathrm{HR}(95 \% \mathrm{Cl})$ & $\begin{array}{l}\text { Days } \\
\text { with SIRS } \geq 2, \mathrm{HR} \\
(95 \% \mathrm{CI})\end{array}$ \\
\hline All patients & $1054(32.9)$ & $5.92(5.03-6.97)$ & $5.05(4.27-5.98)$ \\
\hline Neuromuscular blocking agents with sedatives or opiates ${ }^{\mathrm{a}}$ & $197(97.5)$ & $1.33(0.33-5.37)$ & $1.61(0.39-6.61)$ \\
\hline Sedatives or opiates, without neuromuscular blocking agents ${ }^{a}$ & $720(75.6)$ & $1.90(1.58-2.29)$ & $1.94(1.59-2.36)$ \\
\hline No neuromuscular blocking agents, sedatives, or opiates ${ }^{\mathrm{a}}$ & $137(6.7)$ & $5.08(3.47-7.34)$ & $4.70(3.09-7.15)$ \\
\hline
\end{tabular}

Data reported as $\mathrm{N}(\%)$

$\mathrm{Cl}$ confidence interval, $H R$ hazard ratio, SIRS systemic inflammatory response syndrome

a Medications listed here for stratification of the group include only those give prior to the onset of coma

Table 3 Direct, indirect, and total effects of medications as a mediator in the relationship between SIRS and coma

\begin{tabular}{|c|c|c|c|}
\hline & Estimate & Lower bound & Upper bound \\
\hline \multicolumn{4}{|c|}{ Paralytic medication as mediator } \\
\hline Controlled direct effect & 1.51 & 1.22 & 1.79 \\
\hline Natural direct effect & 1.51 & 1.22 & 1.79 \\
\hline Natural indirect effect & 0.78 & 0.64 & 0.91 \\
\hline Total effect & 2.29 & 2.01 & 2.56 \\
\hline Proportion mediated & 0.60 & 0.58 & 0.62 \\
\hline \multicolumn{4}{|c|}{ Sedative medication as mediator } \\
\hline Controlled direct effect & 0.71 & 0.61 & 0.82 \\
\hline Natural direct effect & 0.71 & 0.61 & 0.82 \\
\hline Natural indirect effect & 0.48 & 0.41 & 0.55 \\
\hline Total effect & 1.19 & 1.07 & 1.32 \\
\hline Proportion mediated & 0.55 & 0.54 & 0.56 \\
\hline \multicolumn{4}{|c|}{ Combined paralytic, sedative, and/or opiate as mediator } \\
\hline Controlled direct effect & 0.51 & 0.42 & 0.60 \\
\hline Natural direct effect & 0.51 & 0.42 & 0.60 \\
\hline Natural indirect effect & 0.41 & 0.35 & 0.46 \\
\hline Total effect & 0.92 & 0.82 & 1.0 \\
\hline Proportion mediated & 0.56 & 0.55 & 0.57 \\
\hline
\end{tabular}

less of an effect on the relationship between SIRS and coma, explaining only $55 \%$ of the relationship between SIRS and coma. Serum markers of inflammation may turn out to define the inflammatory state preceding the onset of coma more precisely, and almost all were found to be elevated preceding coma compared with in patients who did not develop coma (Table S4).

One hundred thirty-seven patients did not receive any neuromuscular blocking agents, sedatives, or opiates prior to the onset of coma (Table 2). Only 3 patients of this cohort of 137 patients did not undergo at least one imaging study, lumbar puncture, or EEG as part of the workup of coma. The large majority of these patients underwent at least one imaging study $(n=133)$; this consisted of a brain computed tomography scan in 116 patient and a brain magnetic resonance imaging scan in 97 patients. As potential causes for coma, imaging studies revealed an ischemic stroke in $12 \%(n=16)$; intracerebral, subarachnoid, or subdural hemorrhages in $6 \%$ $(n=8)$; and imaging findings consistent with posterior reversible leukoencephalopathy in $4 \%(n=5)$ of those who underwent imaging. Importantly, 19\% $(n=26)$ showed no abnormalities, and 59\% $(n=78)$ revealed nonspecific findings, such as white matter hyperintensities, that would not likely explain coma. Additionally, lumbar punctures were obtained in $15 \%(n=21)$, and none of them revealed abnormal findings. EEGs were obtained in three patients, all of whom had nonspecific abnormalities, such as moderate to severe diffuse background slowing $(n=3)$, triphasic waves $(n=1)$, and generalized periodic discharges at $0.5-1 \mathrm{~Hz}(n=1)$, but none revealed seizures.

\section{Outcomes}

Coma was seen in 712 of 2742 (26\%) patients with COVID-19 discharged alive, 329 of 441 (74.6\%) patients who died, and 13 of 20 (65\%) patients still hospitalized. Coma was an independent predictor of death (OR 7.77, 95\% CI 6.29-9.65), after accounting for age, BMI, race/ ethnicity, and sex. The median hospital length of stay was 31 days (IQR 13-59) for patients discharged alive, 16 days (IQR 7-32) for those who died, and 57 days (IQR 48-77) for those who were still hospitalized. The median length of stay was longer for comatose patients compared with those without coma (13 days [IQR 11.9-14.1] vs. 11 [IQR 9.6-12.4]), adjusting for age, BMI, race/ethnicity, and sex.

\section{Discussion}

Impaired consciousness, such as coma, is common in patients hospitalized for COVID-19 and is associated with prolonged hospital stays and death. Prior to the development of coma, vital signs and laboratory changes indicate an increased inflammatory state, with the risk of behavioral abnormalities increasing with each additional 
day that this inflammatory state persisted. This relationship between inflammation and coma was independent of the development of hypoxia and hypotension. The effect was seen for patients who did or did not receive sedative, opioid, or neuromuscular blocking medications, all of which are frequently used in the management of severe COVID-19 disease [1]. Importantly, more than half of these patients did not show any abnormalities on imaging or other diagnostic tests that were ordered to workup the onset of coma. Inflammation and coma have a complex relationship in patients with COVID-19, as ARDS and inflammatory storm may require management with sedatives that predictably impair consciousness. We found evidence that use of sedative, opioid, or neuromuscular blocking medications only accounted for part of the strong relationship between SIRS and coma. This supports that in addition to medication related unconsciousness, inflammation is independently associated with impairment of consciousness. Serum measures of inflammation were elevated preceding the onset of coma (Table S4) but deserve further dedicated investigation. These findings support studies that have implicated inflammation as a contributing factor leading to disorders of consciousness in nonstructural brain injury, such as septic encephalopathy [23] and delirium [24]. Therapeutics considered in the management of patients with COVID-19, for instance remdesivir [25] or IL-6 inhibitors [26], may also have beneficial effects for coma, but this was not systematically explored in the study presented here.

Impaired consciousness is commonly encountered in patients with systemic infections, such as sepsis [23, 27-30]. Early detection of disorders of consciousness is important to direct the diagnostic workup and medical support for these vulnerable patients [31]. However, neurological examinations are challenging in highly contagious patients, such as those encountered during the COVID-19 pandemic. Impairment of consciousness has been reported in $15 \%$ of patients with COVID-19 with severe courses [7]. We were able to obtain behavioral assessments in 3203 patients admitted with COVID-19 acquired over the span of only 5 months from a single hospital in New York City and found that approximately a third of our patients (32\%) were comatose. When interpreting these results, it is important to recognize that patients included in the study were more frequently admitted to the ICU and tended to be older compared with hospitalized patients with COVID-19 who were not included. Even assuming that not a single patient of the 7594 patients without behavioral assessments had coma, $10 \%$ of the 10,797 patients with COVID-19 in the overall cohort were comatose. However, insights gained here should not be generalized to nonhospitalized patients and have to be cautiously interpreted for patients hospitalized with mild to moderate COVID-19. It is difficult to compare prevalence numbers of coma in patients with COVID-19 because triggers for hospital admission, strain of the health care system, institutional management protocols (such as triggers for initiating mechanical ventilation), and presence of medication and medical confounders (such as sedation or renal failure) need to be considered. We present a large hospitalized cohort of patients with COVID-19 that is balanced between ICU and non-ICU patients and chose to compare the effects of medications, medical confounders, and inflammation that precede the onset of coma.

Mechanisms for coma in patients with COVID-19 are poorly understood and likely multifactorial. Inflammatory illnesses have been linked to changes in blood-brain barrier permeability, altered cerebral microcirculation, mitochondrial and vascular endothelial dysfunction, endotoxins and oxidative stress, direct neuronal damage, neurotransmitter disturbances, and changes in amino acid levels that may impair brain function [23]. Prior to the development of coma, vital signs and laboratory changes in our cohort indicate an increased inflammatory state with a rising risk of behavioral abnormalities the longer that this inflammatory state persisted. Intubation and vasopressor support are frequently required for the management of severe COVID-19 [1], but the association between coma and inflammation was independent of hypoxia and hypotension. This observation could be important because the inflammatory response can be tracked using vital signs and laboratory measures and used as a predictor for later behavioral abnormalities. The relationship was independent of sedative or neuromuscular blocking medications, which are frequently used in patients with acute respiratory distress syndrome encountered in severe COVID-19 [1, 32]. Mechanisms for coma in COVID-19 may differ from other conditions, especially given that direct pathogen invasion of the central nervous system, which has been seen in other neurotropic viruses (i.e., Middle East respiratory syndrome-related coronavirus (MERS-CoV)), has not been ruled out with COVID-19 [33]. Promising treatments of COVID-19 may include immunomodulatory approaches $[25,26]$, and beneficial effects for coma should be explicitly tracked.

Coma is well established as a predictor of poor prognosis in septic patients [34], but long-term recovery has been seen for patients with or without acute brain injury [35]. Persistent coma may have a number of causes that are only starting to emerge in patients with COVID-19, including seizures, ischemic and hemorrhagic stroke, and leukoencephalopathy [36]. Many of these patients undergo treatment with deep sedation with continuous 
infusions of sedatives and analgesics and prolonged courses of neuromuscular blockade and have clinical courses complicated by acute kidney injury, leading to the possibility of delayed awakening [1]. Outcomes presented here have to be considered as preliminary, as a sizable portion of this COVID-19 cohort is still hospitalized.

Limitations of our study include the lack of long-term outcomes, a single-center study from the United States, and potential for misclassification. However, given the novelty of COVID-19 disease, we elected to rapidly share these insights with the medical community. We are missing behavioral evaluations in part of the overall cohort of patients admitted with COVID-19 disease, but the large cohort of patients studied here was evenly balanced between hospitalized ICU and non-ICU patients and assessed using highly standardized behavioral assessments [37]. We trust the reliability of our data, but given the challenges encountered during this pandemic, faced with extreme work conditions and overwhelming clinical responsibilities of health care workers, charting inaccuracies have to be considered. It is unlikely, though, that these systematically affected behavioral, medication, or laboratory assessments differentially. Future studies should include more accurate behavioral assessments, such as the Coma Recovery Scale Revised. Our chosen threshold for hypoxia was conservatively set at oxygen saturation $<90 \%$ in an attempt to capture all potential hypoxic events, but this does not adequately explore the impact of hypoxic burden on consciousness. Additionally the analysis of the burden of hypoxia as it relates to the burden of hypotension may yield insights into mechanisms underlying the development of coma and should be explored in future studies. We recognize that sicker patients with COVID-19 have more inflammation; however, this study highlights how a simple measure of systemic inflammation is associated with severe coma. Additionally, we hypothesized that coma is likely driven by sedation use, and performed mediation analyses to account for the role of sedation in the association between inflammation and coma.

\section{Conclusions}

Impaired consciousness, such as coma, is frequent in patients suffering from severe COVID-19 disease. We found that disorders of consciousness in these patients are linked to systemic inflammation, independent of hypoxia and hypotension. These findings may support risk stratification of patients with increased inflammation who are at high risk of developing coma and may have implications for currently employed antiinflammatory treatments.
Supplementary Information

The online version contains supplementary material available at https://doi. org/10.1007/s12028-021-01256-7.

\begin{abstract}
Author details
${ }^{1}$ Department of Epidemiology, Mailman School of Public Health, Columbia University, New York, NY, USA. ${ }^{2}$ Division of Critical Care Neurology, Department of Neurology, Columbia University Irving Medical Center, New YorkPresbyterian Hospital, 177 Fort Washington Avenue, MHB 8 Center, Room 300, New York, NY 10032, USA. ${ }^{3}$ Division of Hospitalist Neurology, Department of Neurology, Columbia University Irving Medical Center, New York-Presbyterian Hospital, New York, NY, USA. ${ }^{4}$ Pharmacy, Columbia University Irving Medical Center, New York-Presbyterian Hospital, New York, NY, USA. ${ }^{5}$ Division of Pulmonary, Allergy, and Critical Care Medicine, Department of Medicine, Columbia University Irving Medical Center, New York-Presbyterian Hospital and Columbia University, New York, NY, USA. ${ }^{6}$ Department of Surgery, Columbia University Irving Medical Center, New York, NY, USA. ${ }^{7}$ Division of Cardiology, Department of Medicine, Columbia University Irving Medical Center, New York, NY, USA. ${ }^{8}$ Division of Critical Care Medicine, Department of Anesthesiology, Columbia University Irving Medical Center, New York, NY, USA.
\end{abstract}

\section{Acknowledgements}

We thank the nurses, respiratory technicians, residents, fellows, and attendings who took care of these patients.

\section{Author Contributions}

$A K B, K D$, and JC conceived and coordinated the study, participated in its design and helped to draft and review the manuscript, contributed to analysis and interpretation of data, and were involved in revising the manuscript. AKB and $K D$ performed the statistical analysis and the data analysis. AKB, KD, JAE, $C D N$, and JC were involved in data preparation and cleaning. AKB, KD, KTT, DR, $\mathrm{SP}, \mathrm{SA}, \mathrm{AGV}, \mathrm{JAE}, \mathrm{CDN}, \mathrm{MLP}, \mathrm{JR}, \mathrm{DB}, \mathrm{KNF}, \mathrm{BRH}, \mathrm{LER}, \mathrm{NHY}, \mathrm{OP}$, and JC contributed to the interpretation of data and were involved in revising the manuscript. All authors meet all authorship requirements of the International Committee of Medical Journal Editors. All authors read and approved the final manuscript.

\section{Data Availability}

All relevant data are presented within the article and its supporting information files. Additional information can be obtained on request to the corresponding author.

\section{Source of Support}

$\mathrm{JC}$ is supported by grant funding from the National Institute of Neurological Disorders and Stroke (R01 NS106014, NS106014-S0201, R01 NS106014-S0202, and R03 NS112760) and by the James McDonnel Foundation.

\section{Conflict of interest}

The authors declare no conflicts of interest.

\section{Ethical Approval/Informed Consent}

This study was approved by the Institutional Review Board at Columbia University Irving Medical Center. The requirement for written informed consent was waived because the observational study design involves no more than minimal risk. The institutional review board approved this study (AAAS9835) on April 3, 2020. The institutional review board approved publication of anonymized data only. The study was conducted according to the rules and regulations of the local the Institutional Review Board at Columbia University Irving Medical Center.

\section{Publisher's Note}

Springer Nature remains neutral with regard to jurisdictional claims in published maps and institutional affiliations.

Received: 4 December 2020 Accepted: 13 April 2021

Published online: 28 June 2021 


\section{References}

1. Cummings MJ, Baldwin MR, Abrams D, et al. Epidemiology, clinical course, and outcomes of critically ill adults with COVID-19 in New York City: a prospective cohort study. Lancet. 2020;395(10239):1763-70.

2. Ellul MA, Benjamin L, Singh B, et al. Neurological associations of COVID19. Lancet Neurol. 2020;19:767-83.

3. Koralnik IJ, Tyler KL. COVID-19: a global threat to the nervous system. Ann Neurol. 2020;88:1-11.

4. Needham EJ, Chou SHY, Coles AJ, Menon DK. Neurological implications of COVID-19 infections. Neurocrit Care. 2020;32:667-71.

5. Paterson RW, Brown RL, Benjamin L, et al. The emerging spectrum of COVID-19 neurology: clinical, radiological and laboratory findings. Brain. 2020;143(10):3104-20.

6. Kotfis K, Williams Roberson S, Wilson JE, et al. COVID-19: ICU delirium management during SARS-CoV-2 pandemic. Crit Care. 2020;24:176.

7. Mao L, Jin H, Wang M, et al. Neurologic manifestations of hospitalized patients with coronavirus disease 2019 in Wuhan, China. JAMA Neurol. 2020;77(6):683-90.

8. Helms J, Kremer S, Merdji H, et al. Neurologic features in severe SARSCoV-2 infection. N Engl J Med. 2020;382:2268-70.

9. Claassen J, Doyle K, Matory A, et al. Detection of brain activation in unresponsive patients with acute brain injury. N Engl J Med. 2019;380:2497-505.

10. Turgeon AF, Lauzier F, Simard J-F, et al. Mortality associated with withdrawal of life-sustaining therapy for patients with severe traumatic brain injury: a Canadian multicentre cohort study. CMAJ. 2011;183:1581-8.

11. Elmer J, Torres C, Aufderheide TP, et al. Association of early withdrawal of life-sustaining therapy for perceived neurological prognosis with mortality after cardiac arrest. Resuscitation. 2016;102:127-35.

12. Claassen J, Albers D, Schmidt JM, et al. Nonconvulsive seizures in subarachnoid hemorrhage link inflammation and outcome. Ann Neurol. 2014;75:771-81.

13. Boehme AK, Kapoor N, Albright KC, et al. Predictors of systemic inflammatory response syndrome in ischemic stroke undergoing systemic thrombolysis with intravenous tissue plasminogen activator. J Stroke Cerebrovasc Dis. 2014;23:e271-6.

14. Boehme AK, Hays AN, Kicielinski KP, et al. Systemic Inflammatory response syndrome and outcomes in intracerebral hemorrhage. Neurocrit Care. 2016;25:133-40.

15. Szklener S, Korchut A, Godek M, et al. Systemic inflammatory response syndrome in the course of status epilepticus: 7-year, two-center observational study. Epilepsy Res. 2017:137:53-5.

16. Hagen M, Sembill JA, Sprügel MI, et al. Systemic inflammatory response syndrome and long-term outcome after intracerebral hemorrhage. Neurol Neuroimmunol Neuroinflamm 2019;6:e588.

17. Boehme AK, Comeau ME, Langefeld CD, et al. Systemic inflammatory response syndrome, infection, and outcome in intracerebral hemorrhage. Neurol Neuroimmunol Neuroinflamm 2017;5:e428.

18. Centers for Disease Control and Prevention. Coronavirus disease 2019 (COVID-19) 2020 interim case definition, approved April 5, 2020. https:// wwwn.cdc.gov/nndss/conditions/coronavirus-disease-2019-covid-19/ case-definition/2020/. Accessed 22 May 2020.
19. Teasdale G, Jennett B. Assessment of coma and impaired. Lancet. 1974;2:81-4.

20. Levy MM, Fink MP, Marshall JC, et al. 2001 SCCM/ESICM/ACCP/ ATS/SIS international sepsis definitions conference. Crit Care Med. 2003:31:1250-6.

21. Valeri L, VanderWeele TJ. Mediation analysis allowing for exposuremediator interactions and causal interpretation: theoretical assumptions and implementation with SAS and SPSS macros. Psychol Methods. 2013;18:137-50.

22. Sepulveda J, Westblade LF, Whittier S, et al. Bacteremia and blood culture utilization during covid-19 surge in New York City. J Clin Microbiol. 2020;58:e00875-e920.

23. Gofton TE, Bryan YG. Sepsis-associated encephalopathy. Nat Rev Neurol. 2012:8:557-66

24. Maldonado JR. Neuropathogenesis of delirium: review of current etiologic theories and common pathways. Am J Geriatr Psychiatry. 2013;21:1190-222.

25. Beigel JH, Tomashek KM, Dodd LE, et al. Remdesivir for the treatment of Covid-19: final report. N Engl J Med. 2020;383(19):1813-26.

26. Mehta P, McAuley DF, Brown M, et al. COVID-19: consider cytokine storm syndromes and immunosuppression. Lancet. 2020;395(10229):1033-4.

27. Cao A, Rohaut B, Le Guennec L, et al. Severe COVID-19-related encephalitis can respond to immunotherapy. Brain. 2020;143:e102.

28. Pilotto A, Masciocchi S, Volonghi l, et al. Clinical presentation and outcomes of severe acute respiratory syndrome coronavirus 2-related encephalitis: the ENCOVID multicenter study. J Infect Dis. 2021;223:28-37.

29. Pilotto A, Masciocchi S, Volonghi I, et al. SARS-CoV-2 encephalitis is a cytokine release syndrome: evidences from cerebrospinal fluid analyses. Clin Infect Dis. 2021. https://doi.org/10.1093/cid/ciaa1933.

30. Perrin P, Collongues N, Baloglu S, et al. Cytokine release syndromeassociated encephalopathy in patients with COVID-19. Eur J Neurol. 2021;28:248-58.

31. Chaudhry N, Duggal AK. Sepsis associated encephalopathy. Adv Med. 2014;2014:762320.

32. Brower RG, Matthay MA, Morris A, et al. Ventilation with lower tidal volumes as compared with traditional tidal volumes for acute lung injury and the acute respiratory distress syndrome. N Engl J Med. 2000:342:1301-8

33. Paniz-Mondolfi A, Bryce C, Grimes Z, et al. Central nervous system involvement by severe acute respiratory syndrome coronavirus-2 (SARSCoV-2). J Med Virol. 2020;92:699-702.

34. Seymour CW, Kahn JM, Cooke CR, et al. Prediction of critical illness during out-of-hospital emergency care. JAMA. 2010;304:747-54.

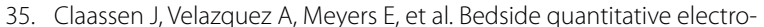
encephalography improves assessment of consciousness in comatose subarachnoid hemorrhage patients. Ann Neurol. 2016;80:541-53.

36. Oxley TJ, Mocco J, Majidi S, et al. Large-vessel stroke as a presenting feature of Covid-19 in the young. N Engl J Med. 2020;382:e60.

37. Reith FCM, Van den Brande R, Synnot A, et al. The reliability of the Glasgow Coma Scale: a systematic review. Intensive Care Med. 2016:42:3-15. 